\title{
Relation between the properties of the kilohertz quasi-periodic oscillations and spectral parameters in $4 \mathrm{U} 1636-53$.
}

\author{
Evandro M. Ribeiro ${ }^{1 \star}$, Mariano Méndez ${ }^{1}$, Guobao Zhang $^{2}$, and Andrea Sanna ${ }^{3}$ \\ ${ }^{1}$ Kapteyn Astronomical Institute, University of Groningen, P.O. BOX 800, 9700 AV Groningen, The Netherlands \\ ${ }^{2}$ New York University Abu Dhabi, P.O. Box 129188, Abu Dhabi, United Arab Emirates \\ ${ }^{3}$ Dipartimento di Fisica, Universitá degli Studi di Cagliari, SP Monserrato-Sestu km 0.7, I-09042, Monserrato, Italy
}

Accepted XXX. Received YYY; in original form ZZZ

\begin{abstract}
We investigate the relation between the parameters of the energy spectrum and the frequency and amplitude of the kilohertz quasi-periodic oscillations (kHz QPOs) in the low-mass X-ray binary $4 \mathrm{U} 1636-53$. We fit the $3-180-\mathrm{keV}$ spectrum of this source with a model that includes a thermal Comptonisation component. We show that the frequencies of both $\mathrm{kHz}$ QPOs follow the same relation as a function of the parameters of this spectral component, except for a systematic frequency shift, whereas the rms fractional amplitude of each QPO follows a different relation with respect to those same parameters. This implies that, while the dynamical mechanism that sets the frequencies of the QPO can be the same for both $\mathrm{kHz}$ QPOs, the radiative mechanisms that set the amplitudes of the lower and the upper $\mathrm{kHz}$ QPO are likely different. We discuss the implications of these results to the modelling of the $\mathrm{kHz}$ QPOs and the possibility that the lower $\mathrm{kHz}$ QPO reflects a resonance between the Comptonising medium and the photons from the accretion disc and/or the neutron star surface.
\end{abstract}

Key words: accretion, accretion discs - stars: neutron - X-rays: binaries - stars: individual: $4 \mathrm{U}$ 1636-53

\section{INTRODUCTION}

The spectral and timing properties of neutron star low-mass $\mathrm{X}$-ray binaries (NS-LMXB) depend upon the position of the source in the so called colour-colour diagram (CD; e.g., Hasinger \& van der Klis 1989). For NS-LMXB the position of the source in the $\mathrm{CD}$ can be described by a single parameter, $S_{a}$ (e.g., Kuulkers et al. 1994; Méndez et al. 1999). The characteristic frequencies observed in the power density spectra (PDS) of these sources are correlated with $S_{a}$ (e.g., Méndez et al. 1999; Van Straaten et al. 2000; Di Salvo et al. 2001, 2003). A typical example is the central frequency of one of the kilohertz quasi-periodic oscillation $(\mathrm{kHz} \mathrm{QPO})$, specifically the upper $\mathrm{kHz} \mathrm{QPO}$, which is positively correlated with $S_{a}$ (Méndez et al. 1999; Belloni et al. 2005, 2007; Sanna et al. 2012).

Hasinger \& van der Klis (1989) proposed that the evolution of the source in the $\mathrm{CD}$ is driven by changes in the mass accretion rate in the system and it is often considered a better tracer of accretion rate than X-ray luminosity (e.g., Kuulkers et al. 1994; Jonker et al. 1998; Méndez et al. 1999;

^ E-mail: ribeiro@astro.rug.nl
Ford et al. 2000; Van Straaten et al. 2000; Méndez et al. 2001). As the accretion rate increases the source spectrum softens due to the increase of the relative contribution of the accretion disc and the neutron star surface to the total emission, and the more efficient cooling of the corona. At the same time, the truncation radius decreases (Done et al. 2007) and the temperature at the inner edge of the disc increases. This scenario is consistent with the correlation between the upper $\mathrm{kHz}$ QPO frequency and $S_{a}$, if this QPO reflects the orbital frequency of matter at the inner edge of the disc (Miller et al. 1998; Stella \& Vietri 1998). The lower $\mathrm{kHz} \mathrm{QPO}$ appears only within a narrow range of $S_{a}$ (e.g., Zhang et al. 2017), and hence a correlation with $S_{a}$ is unclear.

$4 \mathrm{U} 1636-53$ is an LMXB with an orbital period of $3.8 \mathrm{~h}$ (Van Paradijs et al. 1990) and the accreting neutron star has a spin rate of $581 \mathrm{~Hz}$ (Zhang et al. 1997). This source shows a cycle of $\sim 40$ days (Shih et al. 2005) during which it evolves trough different spectral states covering the full CD. This makes it an exceptional target to explore the relation between its timing and spectral properties.

Zhang et al. (2017) recently studied the relation between the presence of the $\mathrm{kHz} \mathrm{QPO}$ and the spectral evolu- 
tion of the source in the NS-LMXB $4 \mathrm{U}$ 1636-53. Based on their results, here we study the relation between the properties of the $\mathrm{kHz} \mathrm{QPO}$ and the spectral properties of this source to gain insight on the mechanism that drives the appearance of the $\mathrm{kHz}$ QPOs. In $\S 2$ we describe the dataset, models and methods used, in $\S 3$ we show our results and, finally, we discuss these results and summarise our findings in $\S 4$

\section{OBSERVATIONS AND DATA ANALYSIS}

For this work we use all 1576 observations of the NSLMXB 4U 1636-53 taken with the Rossi X-ray Timing Explorer (RXTE) Proportional Counter Array (PCA; Jahoda et al. 2006) and the High-Energy X-ray Timing Experiment (HEXTE; Rothschild et al. 1998).

\subsection{Timing Analysis}

We produced Fourier PDS using the event-mode data with a time resolution of $125 \mu$ s covering the full PCA energy band. We set the sample rate to $1 / 4096 \mathrm{~s}$, which yields a Nyquist frequency of $2048 \mathrm{~Hz}$, and calculated a PDS every $16 \mathrm{~s}$; we finally averaged all 16-s PDS of each observation to yield a single PDS per observation.

We ignored frequencies below $200 \mathrm{~Hz}$ and fitted a Lorentzian to each possible QPO in the average PDS of each observation using the same criteria as Sanna et al. (2012), which was to accept all QPOs where the ratio between the Lorentzian normalisation and its negative $1 \sigma$ error was larger than 3 , and the coherence, $\mathrm{Q}$, was larger than 2, where $\mathrm{Q}$ is the ratio between the central frequency and the FWHM of the Lorentzian. When only one $\mathrm{kHz}$ QPO was detected we identified it as upper or lower $\mathrm{kHz}$ QPO based on the branches of the diagram of QPO frequency vs. $S_{a}$ (see, for example, Figure 1 in Sanna et al. 2012). We detected $\mathrm{kHz}$ QPOs in 581 out of 1576 observations; we detected the lower $\mathrm{kHz}$ QPO in 403 and the upper in 206 of those observations.

We then combined the data by averaging the PDS of observations where the mean QPO frequency belongs to the frequency ranges shown in Table 1 , for the lower and upper $\mathrm{kHz}$ QPO, respectively. The choice of these frequency ranges was made to allow an approximately equal number of power spectra per frequency range, except for the two lowest frequency ranges of the lower $\mathrm{kHz} \mathrm{QPO}$, for which there are only a handful of observations available. We did not apply any shift-and-add procedure (Méndez et al. 1998) to combine the power spectra.

For each of the frequency ranges of the lower (upper) QPO we searched the averaged PDS for the corresponding upper (lower) QPO. This allowed us to recover one of the $\mathrm{kHz}$ QPOs from the averaged PDS even if that QPO was not significantly detected in each individual observation.

For each frequency interval of the QPOs we estimated the average background contribution to the PCA lightcurve using the ftool RUNPCABACKEST, and we used this background count rate, together with that of the source, to calculate the rms amplitude of the QPOs for each selection.

\subsection{Spectral Analysis}

For the spectral properties of the source within each frequency interval we used the results from Zhang et al. (2017). To get these parameters Zhang et al. (2017) fitted the PCA and HEXTE spectra of $4 \mathrm{U} 1636-53$ using the package XSPEC v12.7 (Arnaud 1996). To account for interstellar absorption they used the model component PHABs with cross-sections from Balucinska-Church \& McCammon (1992) and solar abundances from Anders \& Grevesse (1989), fixing the column density to $N_{\mathrm{H}}=3.1 \times 10^{21} \mathrm{~cm}^{-2}$ (Sanna et al. 2013). Zhang et al. (2017) added a multiplicative factor to account for calibration uncertainties between the PCA and HEXTE instruments.

The continuum emission of the source was fitted with a model consisting of a multi-colour disc blackbody (DISKBB model, Mitsuda et al. 1984; Makishima et al. 1986), a single temperature blackbody (BBODYRAD) and a Comptonisation model (NTHCOMP, Zdziarski et al. 1996; Życki et al. 1999) to describe, respectively, the emission from the disc, the NS surface (or boundary layer), and the Comptonised component. Zhang et al. (2017) included a Gaussian component with a variable width in the model to account for the presence of an iron line; the energy of the line was fixed to 6.5 $\mathrm{keV}$. The temperature of the disc blackbody at the inner disc radius $\left(k \mathrm{~T}_{\mathrm{dbb}}\right)$ was interpolated along the colour-colour diagram using the joint XMM Newton-RXTE spectral fitting results in Sanna et al. (2013) and set to 0.3, 0.2, 0.4, $0.45,0.6,0.75$ and $0.8 \mathrm{keV}$ for $S_{a}$ equal to $1.1,1.3,1.5,1.7$, $1.9,2.1$, and 2.35 , respectively. They left the temperature of the BBODYRAD component free during the fits.

Further details on the spectral models and the fitting results are given in Zhang et al. (2017). Those authors explored in depth the modelling and interpretation of the $\mathrm{X}$ ray spectra of $4 \mathrm{U} 1636-53$ and their relation to the presence of the lower $\mathrm{kHz}$ QPO. For this work we use the spectral parameters found by Zhang et al. (2017) to represent the best-fitting model for each observation.

From the Comptonisation model, assuming a spherically symmetric region, the asymptotic power-law photon index, $\Gamma$, can be expressed as:

$\Gamma=\left[\frac{9}{4}+\frac{1}{\left(k T_{e} / m_{e} c^{2}\right) \tau(1+\tau / 3)}\right]^{1 / 2}-\frac{1}{2}$,

where $\tau$ is the optical depth of the region, and $k T_{e}$ is the electron temperature (Sunyaev \& Titarchuk 1980). Both $\Gamma$ and $k T_{e}$ are obtained from the best fitting model, and are used to calculate the optical depth $\tau$ from Equation 1.

We excluded observations with power-law index $\Gamma<1.2$ for the averaging of spectral parameters, as that is close to the lower boundary of the allowed parameter values $(\Gamma=1)$ and represents a possible unconstrained fit (Zhang et al. 2017). That left us with a total of 558 observations, containing 385 observations with a lower $\mathrm{kHz}$ QPO and 201 with an upper $\mathrm{kHz}$ QPO.

In order to parameterize the spectral evolution of the source we extracted the PCA lightcurves in 4 energy-bands, following Zhang et al. (2017), to create a colour-colour diagram (Fig. 1). The hard and soft colours were defined as the count rate ratio between the energy bands 9.7-16.0/6.0-9.7 
Table 1. Overview of the selection by QPO frequencies used in this work and, for each frequency interval, the average spectral parameters taken from Zhang et al. (2017), and the fractional rms amplitude of the QPO. The numbers in between parentheses represent the number of observations used to investigate timing-only properties, before excluding the ones with low values of $\Gamma$ (see text). Uncertainties in the rms fractional amplitude represent the $1 \sigma$ confidence interval obtained from the best fit to the combined power spectra. Uncertainties in the other variables represent the $1 \sigma$ error of the mean in each averaged interval.

\begin{tabular}{|c|c|c|c|c|c|c|c|}
\hline \multicolumn{8}{|c|}{ Lower kHz QPO } \\
\hline $\begin{array}{l}\text { Frequency } \\
\text { range }(\mathrm{Hz})\end{array}$ & $\begin{array}{l}\text { Number of } \\
\text { observations }\end{array}$ & $\begin{array}{l}\text { Average } \\
\text { frequency }(\mathrm{Hz})\end{array}$ & $S_{a}$ & $\Gamma$ & $k T_{e}(\mathrm{keV})$ & $\tau$ & rms (\%) \\
\hline $470-590$ & 4 & $562.1 \pm 8.4$ & $2.013 \pm 0.039$ & $2.13 \pm 0.04$ & $4.26 \pm 0.46$ & $7.6 \pm 0.7$ & $4.27 \pm 0.30$ \\
\hline $590-620$ & 4 & $608.1 \pm 6.3$ & $2.000 \pm 0.013$ & $2.12 \pm 0.07$ & $4.79 \pm 0.49$ & $7.1 \pm 0.6$ & $4.49 \pm 0.50$ \\
\hline $620-670$ & $28(30)$ & $648.1 \pm 2.3$ & $2.060 \pm 0.005$ & $2.13 \pm 0.05$ & $4.64 \pm 0.30$ & $7.8 \pm 0.6$ & $6.55 \pm 0.15$ \\
\hline $670-715$ & $35(38)$ & $696.6 \pm 2.1$ & $2.093 \pm 0.005$ & $2.05 \pm 0.04$ & $3.86 \pm 0.13$ & $9.1 \pm 0.7$ & $7.56 \pm 0.14$ \\
\hline $715-750$ & $36(37)$ & $733.0 \pm 2.0$ & $2.096 \pm 0.004$ & $2.07 \pm 0.05$ & $3.68 \pm 0.13$ & $9.4 \pm 0.7$ & $7.95 \pm 0.13$ \\
\hline $750-790$ & $39(42)$ & $766.8 \pm 1.7$ & $2.111 \pm 0.004$ & $1.98 \pm 0.04$ & $3.21 \pm 0.08$ & $10.3 \pm 0.4$ & $7.62 \pm 0.10$ \\
\hline $790-820$ & $40(42)$ & $806.6 \pm 1.2$ & $2.118 \pm 0.005$ & $1.90 \pm 0.04$ & $3.09 \pm 0.09$ & $11.2 \pm 0.5$ & $7.74 \pm 0.09$ \\
\hline $820-850$ & $45(46)$ & $834.5 \pm 1.5$ & $2.133 \pm 0.005$ & $1.93 \pm 0.03$ & $3.03 \pm 0.07$ & $10.9 \pm 0.4$ & $7.65 \pm 0.06$ \\
\hline $850-880$ & $55(59)$ & $864.1 \pm 1.2$ & $2.134 \pm 0.004$ & $1.81 \pm 0.03$ & $2.81 \pm 0.04$ & $12.4 \pm 0.4$ & $6.57 \pm 0.05$ \\
\hline $880-910$ & $55(57)$ & $895.7 \pm 1.2$ & $2.142 \pm 0.005$ & $1.77 \pm 0.03$ & $2.78 \pm 0.03$ & $13.1 \pm 0.5$ & $5.48 \pm 0.06$ \\
\hline $910-975$ & 44 & $922.5 \pm 1.3$ & $2.149 \pm 0.004$ & $1.81 \pm 0.03$ & $2.85 \pm 0.05$ & $12.4 \pm 0.4$ & $3.98 \pm 0.08$ \\
\hline \multicolumn{8}{|c|}{ Upper kHz QPO } \\
\hline $440-540$ & 12 & $490.2 \pm 9.8$ & $1.336 \pm 0.022$ & $1.80 \pm 0.01$ & $9.53 \pm 0.34$ & $6.0 \pm 0.2$ & $13.5 \pm 0.7$ \\
\hline $540-650$ & 27 & $607.4 \pm 4.9$ & $1.509 \pm 0.017$ & $1.90 \pm 0.01$ & $12.28 \pm 2.47$ & $5.5 \pm 0.3$ & $11.9 \pm 0.5$ \\
\hline $650-750$ & 32 & $704.9 \pm 4.2$ & $1.710 \pm 0.009$ & $2.01 \pm 0.02$ & $10.46 \pm 2.01$ & $5.5 \pm 0.2$ & $11.5 \pm 0.3$ \\
\hline $750-810$ & 42 & $783.0 \pm 2.4$ & $1.837 \pm 0.006$ & $2.14 \pm 0.02$ & $10.13 \pm 1.82$ & $5.3 \pm 0.2$ & $11.4 \pm 0.2$ \\
\hline $810-870$ & 28 & $839.5 \pm 3.4$ & $1.918 \pm 0.005$ & $2.17 \pm 0.02$ & $7.03 \pm 0.79$ & $5.9 \pm 0.3$ & $10.5 \pm 0.2$ \\
\hline $870-930$ & $18(20)$ & $896.5 \pm 4.4$ & $1.989 \pm 0.006$ & $2.19 \pm 0.03$ & $5.13 \pm 0.38$ & $6.7 \pm 0.4$ & $9.1 \pm 0.2$ \\
\hline $930-1025$ & 17 & $970.0 \pm 6.6$ & $2.054 \pm 0.009$ & $2.09 \pm 0.06$ & $4.34 \pm 0.27$ & $8.3 \pm 0.9$ & $6.5 \pm 0.3$ \\
\hline $1025-1165$ & 7 & $1127.5 \pm 13.2$ & $2.160 \pm 0.015$ & $1.79 \pm 0.06$ & $2.75 \pm 0.03$ & $12.6 \pm 1.0$ & $3.5 \pm 0.3$ \\
\hline $1165-1250$ & $18(21)$ & $1204.8 \pm 4.3$ & $2.194 \pm 0.014$ & $1.76 \pm 0.05$ & $2.79 \pm 0.03$ & $13.3 \pm 1.0$ & $2.6 \pm 0.2$ \\
\hline
\end{tabular}

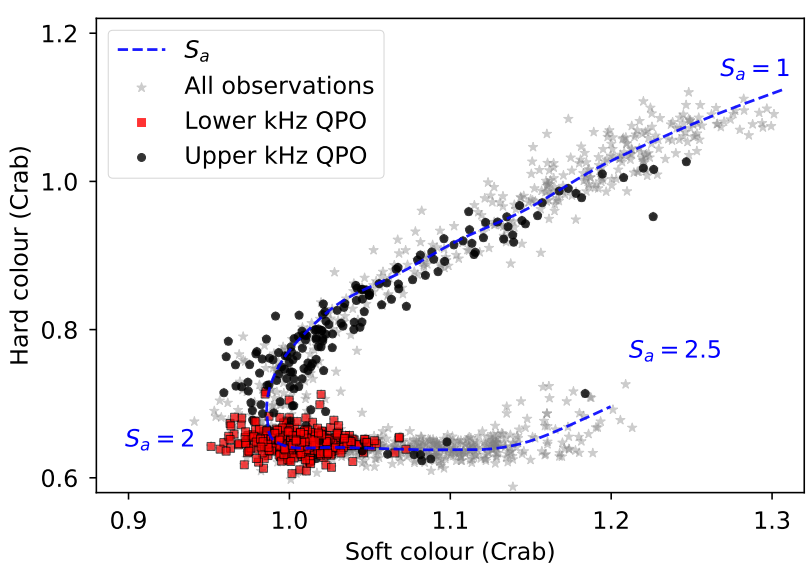

Figure 1. Colour-colour diagram of $4 \mathrm{U} 1636-53$. Each point in this diagram represents a full RXTE/PCA observation. Red and black points represent observations with, respectively, a lower or an upper $\mathrm{kHz}$ QPO. (Observations with two simultaneous $\mathrm{kHz}$ QPOs are shown in red.) Grey points represent observations without $\mathrm{kHz}$ QPOs

$\mathrm{keV}$ and 3.5-6.0/2.0-3.5 keV, respectively, per observation. The parameterization, $S_{a}$, is defined by the length of the dashed-blue line in Fig. 1, fixing $S_{a}=1$ at the top right position of the diagram and $S_{a}=2$ at the bottom left position (e.g. Méndez et al. 1999).

\section{RESULTS}

For each of the frequency intervals of the QPO defined in Table 1, we computed the averaged spectral parameters of the source and we investigated the relation between these averaged parameters and the averaged QPO properties. In Fig. 2 we show the relation between averaged spectral parameters and the frequency of the $\mathrm{kHz}$ QPOs. In all panels red and black (grey and black in the printed version) symbols represent the lower and upper $\mathrm{kHz}$ QPO, respectively.

In the upper-left panel of Fig. 2 we show the power-law index, $\Gamma$, as a function of QPO frequency. It is apparent that $\Gamma$ decreases from $\sim 2.2$ to $\sim 1.8$ as the frequency of the lower $\mathrm{kHz}$ QPO increases from $\sim 500 \mathrm{~Hz}$ to $\sim 900 \mathrm{~Hz}$, while for the upper $\mathrm{kHz}$ QPO $\Gamma$ first increases from $\sim 1.8$ to $\sim 2.2$ as the frequency of this QPO increases from $\sim 500 \mathrm{~Hz}$ to $\sim 850$ $\mathrm{Hz}$, and then decreases from $\sim 2.2$ to $\sim 1.8$ as the frequency of this QPO increases to approximately $1200 \mathrm{~Hz}$.

In the middle-left panel of Fig. 2 we show the relation between the optical depth, $\tau$, and the frequency of the $\mathrm{kHz}$ QPOs. This figure shows that $\tau$ increases from 6 to 14 as the frequency of the lower $\mathrm{kHz}$ QPO increases from $\sim 500 \mathrm{~Hz}$ to $\sim 900 \mathrm{~Hz}$. As the frequency of the upper $\mathrm{kHz}$ QPO increases from $500 \mathrm{~Hz}$ to $850 \mathrm{~Hz} \tau$ remains approximately constant between 5 and 6 and then increases to 14 as the frequency increases from $800 \mathrm{~Hz}$ to $1200 \mathrm{~Hz}$.

On the bottom-left panel of Fig. 2 we show the electron temperature of the Comptonising region, $k T_{e}$, as a function of QPO frequency. The temperature remains approximately constant as the frequency of the lower $\mathrm{kHz}$ QPO increases from $500 \mathrm{~Hz}$ to $600 \mathrm{~Hz}$ and decreases slowly to $3 \mathrm{keV}$ as 


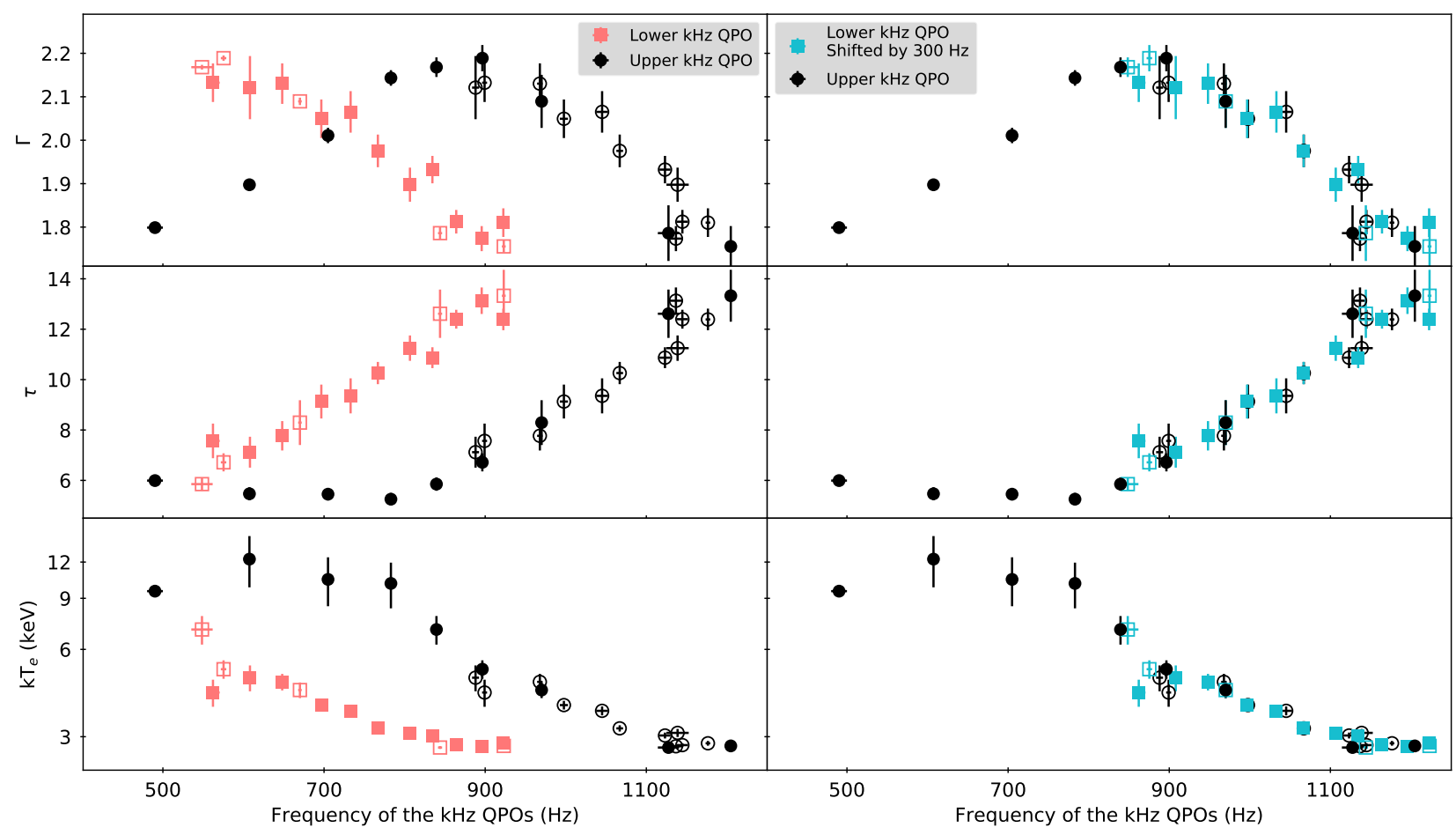

Figure 2. Relation between spectral parameters and $\mathrm{kHz}$ QPO frequency in $4 \mathrm{U}$ 1636-53. The data are the average in the frequency ranges given in section 2. For the plots on the right side the frequency of the lower $\mathrm{kHz}$ QPO was shifted by $300 \mathrm{~Hz}$, which is close to the average separation between the twin $\mathrm{kHz}$ QPO (Jonker et al. 2002). The open black (red or blue) symbols (respectively black and grey in the printed version) correspond to the upper (lower) $\mathrm{kHz}$ QPO detected after combining the PDSs within the frequency ranges chosen for the lower (upper) $\mathrm{kHz}$ QPO. Error bars represent the $1 \sigma$ error of the mean in each bin. The $y$ axis is shown in log scale in the two bottom panels for better visualisation.

the frequency increases to $900 \mathrm{~Hz}$. As the frequency of the upper $\mathrm{kHz}$ QPO increases from $400 \mathrm{~Hz}$ to $600 \mathrm{~Hz} k T_{e}$ remains approximately constant, and then decreases slowly to $3 \mathrm{keV}$ as the QPO frequency increases to $1200 \mathrm{~Hz}$

The right-side panels of Fig. 2 reproduce the left-side plots but with the frequency of the lower $\mathrm{kHz}$ QPO shifted by $300 \mathrm{~Hz}$, which is close to the average separation between the upper and lower $\mathrm{kHz}$ QPO frequencies (Jonker et al. 2002). These panels show that the relation between the spectral parameters and the frequency of the upper $\mathrm{kHz}$ QPO and the shifted lower $\mathrm{kHz}$ QPO can be described by a single relation, as expected due to the almost constant frequency separation between both $\mathrm{kHz}$ QPOs. These plots also show that the relations are valid for each QPO regardless of the presence of the other QPO.

In Fig. 3 we show the parameter $S_{a}{ }^{1}$ and the fractional rms amplitude of the QPOs as a function of $\tau$ and $k T_{e}$. In the top-left panel of Fig. 3 we show that as $S_{a}$ increases from $\sim 1.2$ to $\sim 1.8$ the optical depth $\tau$ of the corona remains approximately constant, and then $\tau$ increases steeply as $S_{a}$ increases from $\sim 1.8$ to $\sim 2.2$. As noticed by Zhang et al. (2017) the lower $\mathrm{kHz}$ QPO is detected only when $\tau$ is larger

1 As explained in $\S 1, S_{a}$ is an indicator of the state of the source and hence it is usually displayed in the $x$-axis but here we choose not to follow this convention in order to share both $x$ and $y$ axis for adjacent panels, allowing for a quick comparison between the different relations. than $\sim 6$ and increases rapidly. The relation between $S_{a}$ and electron temperature $k T_{e}$ is shown in the top-right panel. As $S_{a}$ increases from $\sim 1.2$ to $\sim 1.8$ the temperature is consistent with being constant within errors, and decreases abruptly as $S_{a}$ increases from $\sim 1.8$ to $\sim 2.2$. This trend is similar to the one in the top-left panel of this Figure, but one has to keep in mind that the optical depth is obtained from the fitted values of $\Gamma$ and $k T_{e}$.

The bottom-left panel of Fig. 3 shows the fractional rms amplitude of both QPOs as a function of the optical depth $\tau$ of the Comptonising component. When $\tau$ is low $(<7)$ the amplitude of the upper $\mathrm{kHz}$ QPO is high $(>10 \%)$ and the amplitude decreases to a minimum as $\tau$ increases to $\sim 11$. The lower $\mathrm{kHz}$ QPO displays a very different trend with its rms amplitude increasing from $\sim 4 \%$ to $\sim 8 \%$ as $\tau$ increases from $\sim 5$ to $\sim 11$, and then decreasing as $\tau$ continues increasing to $\sim 14$

We observe a similar trend in the bottom-right panel showing the relation of the rms amplitude of each QPO with the electron temperature of the Comptonising region. The amplitude of the upper $\mathrm{kHz}$ QPO is low $(\sim 4 \%)$ when the temperature is below $3 \mathrm{keV}$ and it increases up to $\sim 14 \%$ as the temperature raises to $\sim 15 \mathrm{keV}$. Meanwhile the amplitude of the lower $\mathrm{kHz}$ QPO increases from $3 \%$ to $8 \%$ as the temperature increases from $\sim 2.5 \mathrm{keV}$ to $\sim 3.5 \mathrm{keV}$ and then decreases to $\sim 5 \%$ as the temperature increases up to $\sim 7$ $\mathrm{keV}$.

Fig. 4 shows the rms amplitude vs. frequency of both 


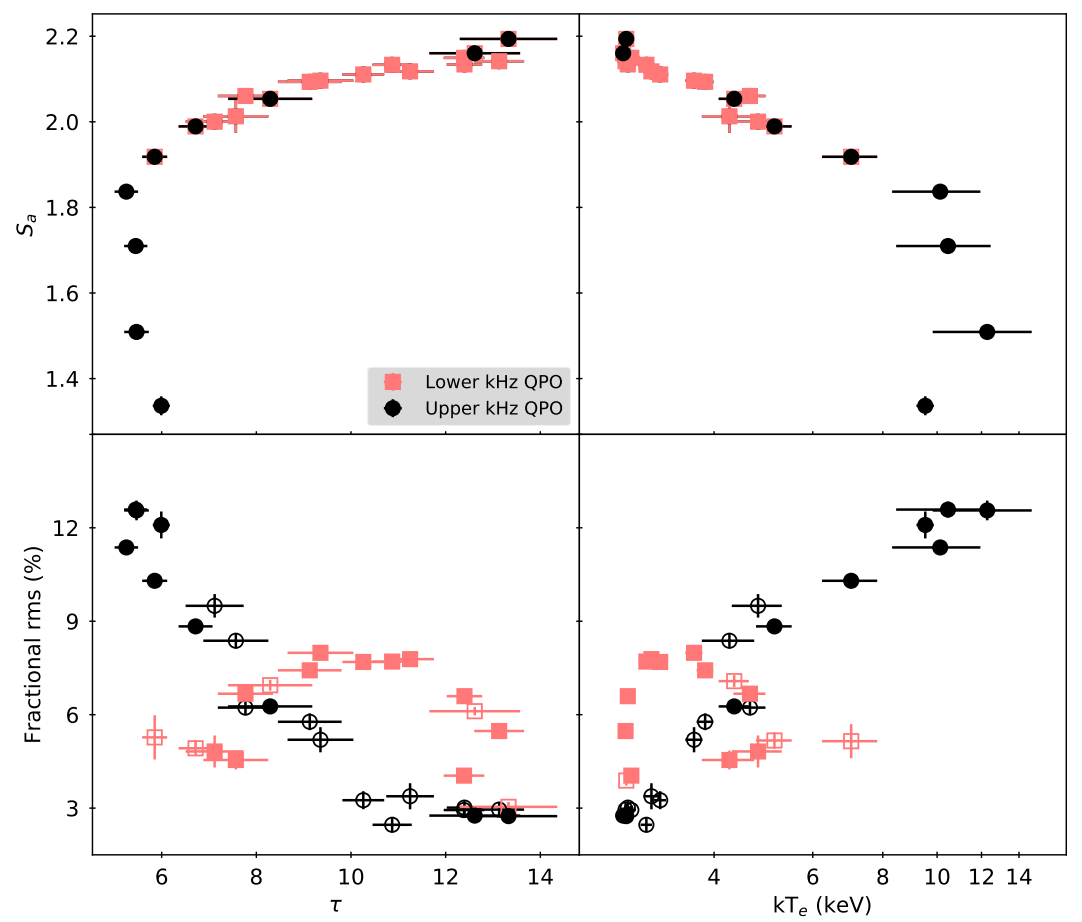

Figure 3. Relations of $S_{a}$ and fractional rms amplitude as a function of spectral parameters in $4 \mathrm{U}$ 1636-53. Symbols are the same as in Fig. 2. The $x$ axis of the right side panels are shown in $\log$ scale for better visualisation. Error bars in rms represent the $1 \sigma$ confidence interval obtained from the best fit to the combined power spectra. Error bars in the other variables represent the $1 \sigma$ error of the mean in each bin.

kHz QPO (see, for example, Méndez et al. 2001; Di Salvo et al. 2003; Barret et al. 2005b; Méndez 2006; Altamirano et al. 2008; Boutelier et al. 2010). The amplitude of the upper QPO decreases from approximately $15 \%$ to $2 \%$ as its frequency increases, with a local maximum $(\sim 11 \%)$ around $800 \mathrm{~Hz}$, while the amplitude of the lower QPO increases from $4 \%$ to $8 \%$ as its frequency increases from approximately 500 $\mathrm{Hz}$ to $800 \mathrm{~Hz}$ and then decreases to $4 \%$ as the frequency increases to $950 \mathrm{~Hz}$.

To quantify the local maximum observed in the plot of the rms amplitude vs. frequency for the upper $\mathrm{kHz} \mathrm{QPO}$, we first fitted the data points with a linear function (Model 1) and then with a model consisting of a linear function and a Gaussian (Model 2). We give the best fitting parameters in Table 2 and we plot the data and best-fitting models in Fig. 4.

Based on Model 1 we simulated $10^{7}$ datasets with points drawn from a normal distribution with $\sigma$ equal to the $1 \sigma$ uncertainties of the rms amplitude at each frequency. We fitted all $10^{7}$ realisations with Model 2 with the FWHM and central frequency of the Gaussian fixed to the best-fitting values, leaving the amplitude of the Gaussian free. From all $10^{7}$ there was no realisation with an amplitude equal or larger than the one we obtained in Model 2, which implies a probability smaller than $10^{-7}$ that the observed local maximum arises from statistical fluctuations of data that follow a linear relation.

For completeness, and to find the QPO frequency at which the fractional rms amplitude is maximum, we also fitted a Gaussian function to the amplitude of the lower

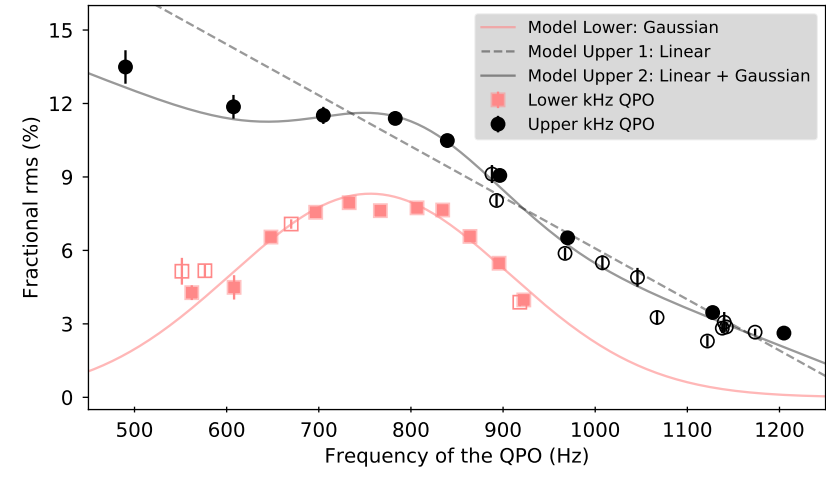

Figure 4. The rms amplitude of the upper and lower $\mathrm{kHz}$ QPO in $4 \mathrm{U} 1636-53$ as a function of the QPO frequency. Symbols are the same as in Fig. 2. The dashed-black and solid-black lines show, respectively, the best fitting linear function and linear function plus a Gaussian to the rms amplitude of the upper $\mathrm{kHz}$ QPO. The solid-red (grey in the printed version) line shows the best fitting Gaussian function to the rms amplitude of the lower $\mathrm{kHz}$ QPO.

$\mathrm{kHz}$ QPO. We show the best-fitting parameters in Table 2, and we plot the data and the model in Fig. 4. Our choice of models has no theoretical support and was made simply as a way to quantitatively describe the shape of the rms amplitude of the $\mathrm{kHz}$ QPOs as a function of QPO frequency. The errors of the parameters shown in Table 2 are likely 
Table 2. Best fitting parameters for the rms amplitude of the $\mathrm{kHz}$ QPOs in $4 \mathrm{U} 1636-53$ as a function of the QPO frequency

\begin{tabular}{lll}
\hline Upper kHz QPO & $\begin{array}{l}\text { Model 1: } \\
\text { Linear }\end{array}$ & $\begin{array}{l}\text { Model 2: } \\
\text { Linear }+ \text { Gaussian }\end{array}$ \\
\hline Constant term (\%) & $26.9 \pm 0.3$ & $19 \pm 1$ \\
Slope $\left(\% \mathrm{~Hz}^{-1}\right)$ & $-0.0208 \pm 0.0003$ & $-0.0148 \pm 0.0008$ \\
Central frequency (Hz) & - & $799 \pm 11$ \\
FWHM (Hz) & - & $229 \pm 29$ \\
Amplitude (\%) & - & $3.2 \pm 0.3$ \\
$\chi^{2} /$ d.o.f & $215.5 / 18$ & $65.6 / 15$ \\
\hline Lower kHz QPO & & \\
& Model: & \\
& Gaussian & \\
\hline Central frequency (Hz) & $755.9 \pm 1.5$ & \\
FWHM (Hz) & $355 \pm 5$ & \\
Amplitude (\%) & $8.32 \pm 0.05$ & \\
$\chi^{2} /$ d.o.f & $202.9 / 12$ & \\
\hline
\end{tabular}

underestimated given that the model is phenomenological and the fits are statistically unacceptable.

\section{DISCUSSION}

We show that in the NS-LMXB $4 \mathrm{U} 1636-53$ the frequency of the lower and upper $\mathrm{kHz}$ QPOs follow the same relation, albeit shifted by $\sim 300 \mathrm{~Hz}$ from each other, as a function of the spectral parameters of the source, but the fractional amplitude of the lower and upper $\mathrm{kHz}$ QPOs follow completely different relations. In the latter case the relation of the rms of one QPO cannot be shifted to obtain the relation of the other.

For frequencies below $\sim 400 \mathrm{~Hz}$ we do not detect the $\mathrm{kHz}$ QPOs, as the PDS of the source starts featuring hectohertz QPOs and broad-band noise components (see Altamirano et al. 2008 for an overview of the X-ray variability in this source). Therefore it is not possible to tell if Figures 2 and 3 represent the whole picture. For example, one might question whether the lower $\mathrm{kHz} \mathrm{QPO}$ is never present when the temperature $k T_{e}$ is above $8 \mathrm{keV}$, or whether it is simply not detected because its frequency would lie in the noisy region of the PDS.

The frequency of the upper $\mathrm{kHz}$ QPO is generally associated with the Keplerian motion of matter at the innermost parts of the accretion disc (e.g. Miller et al. 1998; Stella \& Vietri 1998; Lamb \& Miller 2001; Török et al. 2016); this idea is consistent with the relation between QPO frequency and $S_{a}$, which can be understood in terms of changes in the radius at which the QPO is produced due to changes in mass accretion rate (see $\S 1$ ).

Fig. 2 shows that the dynamical mechanisms that set the frequency of both $\mathrm{kHz}$ QPOs are strongly related, or are possibly the same. The frequency of the lower $\mathrm{kHz}$ QPO can be shifted to obtain a single relation between the spectral parameters that describe the Comptonising component of the energy spectrum and the $\mathrm{kHz}$ QPOs frequencies. Considering that we observe the upper $\mathrm{kHz}$ QPO in different spectral states, and the frequencies of both the upper and lower $\mathrm{kHz}$ QPO have the same dependence with spectral parameters, we can argue that, at least dynamically, both $\mathrm{kHz}$ QPOs should always be present. The fact that we do not always detect these QPOs in pairs may be due to differences in the radiative mechanisms that set their amplitudes.

In contrast to the behaviour of the frequencies shown in Fig. 2, Fig. 3 shows that the rms fractional amplitude of the upper and lower $\mathrm{kHz}$ QPOs have disparate behaviours as a function of spectral parameters. In this case, it is not possible to shift the frequencies or amplitudes of one QPO to obtain the relation shown by the other. The amplitude of the lower $\mathrm{kHz}$ QPO shows a maximum for a specific range of values of the spectral parameters and QPO frequency, whereas the amplitude of the upper $\mathrm{kHz}$ QPO decreases more or less monotonically with frequency, with a local maximum (seen as a hump in Fig. 4) at $\sim 800 \mathrm{~Hz}$. Therefore the radiative mechanisms that sets the amplitude of, respectively, the lower and the upper $\mathrm{kHz} \mathrm{QPO}$ are likely different. It is interesting to notice that the energy and frequency dependence of the time lags of the $\mathrm{kHz}$ QPOs in $4 \mathrm{U} 1636-53$ also suggest two different radiative mechanisms responsible for each QPO (De Avellar et al. 2013).

As Zhang et al. (2017) pointed out, at $\sim 850 \mathrm{~Hz}$ the lower $\mathrm{kHz}$ is more often detected and has its highest quality factor (Belloni et al. 2005; Barret et al. 2006); this is also the frequency at which the phase lags of the lower $\mathrm{kHz}$ QPO are the largest and the coherence between low- and high-energy signals is the highest (De Avellar et al. 2013, 2016). All these phenomena happening over a relatively narrow range of frequencies, and specific spectral parameters, suggest that a resonance mechanism may drive the amplitude of the lower $\mathrm{kHz}$ QPO.

We fitted the rms amplitude of both $\mathrm{kHz}$ QPO in order to describe the shape of the rms as a function of the QPO frequency. We find that the local maximum of the amplitude of the upper $\mathrm{kHz}$ QPOs and the global maximum of the amplitude of the lower $\mathrm{kHz}$ QPO occur respectively at $800 \mathrm{~Hz}$ and $\sim 760 \mathrm{~Hz}$. Those values were obtained by fitting simple mathematical models to the rms amplitude and they represent the central values of broad features of the rmsfrequency relation.

While this may only be a coincidence, it is curious that the peaks of these features are at similar frequencies. (Notice that the spectral properties of the source are different when the lower $\mathrm{kHz}$ QPO is at $800 \mathrm{~Hz}$ than when the upper $\mathrm{kHz}$ QPO is at that same frequency.) It could be that a specific combination of spectral parameters (and size, which we do not measure in this work) of the corona triggers (or amplifies) the coupling between disc and corona. One possible scenario is that there is a radiative mechanism that sets the rms amplitude of both QPOs, which peaks at $\sim 800 \mathrm{~Hz}$, whereas there is another mechanism that acts only upon the upper $\mathrm{kHz}$ QPO and drives the more or less linear decay of its rms with frequency. (Notice that this does not necessarily implies that the QPO frequency drives the rms amplitude, but the rms amplitude could also be determined by the spectral properties of the source.)

The amplitude of both $\mathrm{kHz}$ QPOs increases with photon energy (e.g., Berger et al. 1996; Wijnands et al. 1997; Méndez et al. 2001; Gilfanov et al. 2003); together with our results this reinforces the notion that even if the oscillations are driven by the dynamics of matter at particular radii in 
the accretion disc, as for example at the innermost stable circular orbit (e.g, Miller et al. 1998; Lamb \& Miller 2001; Barret et al. 2005a,b, 2006, 2007), the amplitudes of the QPOs are driven by changes in the spectral component that is responsible for the high energy emission in the energy spectrum. Another possibility is that the oscillations arise from the high-energy component itself (Lee et al. 2001; see also Kumar \& Misra 2014), where the Comptonising plasma oscillates at the lower $\mathrm{kHz}$ QPO frequency or, as proposed by Gilfanov et al. (2003), the QPOs reflect oscillations in a Comptonised boundary layer.

Our results support the idea that the properties of the $\mathrm{kHz}$ QPOs are the outcome of a complex interplay between the dynamics of matter around the neutron star, and the radiative processes and the interactions between the accretion disc, the neutron star surface, and the Comptonising region. The fact that frequency and amplitude of the $\mathrm{kHz}$ QPO have different behaviours in relation to the spectral parameters should be inherent to any physical model proposed to describe the mechanisms that produce each of the $\mathrm{kHz}$ QPOs in 4U 1636-53, and possibly other sources.

A key step to understand the nature of the mechanism behind the $\mathrm{kHz}$ QPOs in NS-LMXB is the combined use of spectral and timing techniques to find the link between the timing properties and the physical state of the systems. We gave here the first steps in this direction by comparing the properties of the $\mathrm{kHz} \mathrm{QPO}$ and the parameters that describe the Comptonising region on $4 \mathrm{U}$ 1636-53. Our results should help the development of models that would allow us to describe the physical properties of the Comptonising region, the neutron star itself, and the accretion flow around it, by using the timing properties of the $\mathrm{kHz}$ QPO.

\section{ACKNOWLEDGEMENTS}

The authors are grateful to the anonymous referee for contributing to the improvement of this paper. E.M.R acknowledges the support from Conselho Nacional de Desenvolvimento Científico e Tecnológico ( $\mathrm{CNPq}$ - Brazil). This research has made use of data obtained from the High Energy Astrophysics Science Archive Research Center (HEASARC), provided by NASAs Goddard Space Flight Center

\section{REFERENCES}

Altamirano D., van der Klis M., Méndez M., Jonker P. G., KleinWolt M., Lewin W. H. G., 2008, ApJ, 685, 436

Anders E., Grevesse N., 1989, Geochimica Cosmochimica Acta, 53, 197

Arnaud K., 1996, Astronomical Data Analysis Software and Systems V, 101, 17

Balucinska-Church M., McCammon D., 1992, ApJ, 400, 699

Barret D., Olive J.-F., Miller M. C., 2005a, Astron. Nachr., 326, 808

Barret D., Olive J.-F., Miller M. C., 2005b, MNRAS, 361, 855

Barret D., Olive J. F., Miller M. C., 2006, MNRAS, 370, 1140

Barret D., Olive J. F., Miller M. C., 2007, MNRAS, 376, 1139

Belloni T., Méndez M., Homan J., 2005, A\&A, 437, 209

Belloni T. M., Homan J., Motta S., Ratti E., Méndez M., 2007, MNRAS, 379, 247

Berger M., et al., 1996, ApJ, 469, L13
Boutelier M., Barret D., Lin Y., Török G., 2010, MNRAS, 401, 1290

De Avellar M. G. B., Méndez M., Sanna A., Horvath J. E., 2013, MNRAS, 433, 3453

De Avellar M. G. B., Méndez M., Altamirano D., Sanna A., Zhang G., 2016, MNRAS, 461, 79

Di Salvo T., Méndez M., van der Klis M., Ford E., Robba N. R., 2001, ApJ, 546, 1107

Di Salvo T., Méndez M., van der Klis M., 2003, A\&A, 192, 17

Done C., Gierliński M., Kubota A., 2007, A\&ARv, 15, 1

Ford E. C., van der Klis M., Mendez M., Wijnands R., Homan J., Jonker P. G., van Paradijs J., 2000, ApJ, 537, 368

Gilfanov M., Revnivtsev M., Molkov S., 2003, A\&A, 410, 217

Hasinger G., van der Klis M., 1989, A\&A, 225, 79

Jahoda K., Markwardt C. B., Radeva Y., Rots A. H., Stark M. J., Swank J. H., Strohmayer T. E., Zhang W., 2006, ApJS, 163, 401

Jonker P. G., Wijnands R., van der Klis M., Psaltis D., Kuulkers E., Lamb F. K., 1998, ApJ, 499, L191

Jonker P. G., Méndez M., van der Klis M., 2002, MNRAS, 336, 1996

Kumar N., Misra R., 2014, MNRAS, 445, 2818

Kuulkers E., van der Klis M., Oosterbroek T., Asai K., Dotani T., van Paradijs J., Lewin W. H. G., 1994, A\&A, 289, 795

Lamb F. K., Miller M. C., 2001, ApJ, 554, 1210

Lee H. C., Misra R., Taam R. E., 2001, ApJ, 549, L229

Makishima K., Maejima Y., Mitsuda K., Bradt H. V., Remillard R. A., Tuohy I. R., Hoshi R., Nakagawa M., 1986, ApJ, 308, 635

Méndez M., 2006, MNRAS, 371, 1925

Méndez M., et al., 1998, ApJ, 494, L65

Méndez M., van der Klis M., Ford E. C., Wijnands R. a. D., van Paradijs J., 1999, ApJ, 511, L49

Méndez M., van der Klis M., Ford E. C., 2001, ApJ, 561, 1016

Miller M. C., Lamb F. K., Psaltis D., 1998, ApJ, 508, 791

Mitsuda K., et al., 1984, PASJ, 36, 741

Rothschild R. E., et al., 1998, ApJ, 496, 538

Sanna A., Méndez M., Belloni T. M., Altamirano D., 2012, MNRAS, 424, 2936

Sanna A., Hiemstra B., Méndez M., Altamirano D., Belloni T., Linares M., 2013, MNRAS, 432, 1144

Shih I. C., Bird A. J., Charles P. A., Cornelisse R., Tiramani D., 2005, MNRAS, 361, 602

Stella L., Vietri M., 1998, ApJ, 492, L59

Sunyaev R. a., Titarchuk L. G., 1980, A\&A, 86, 121

Török G., Goluchová K., Horák J., Šrámková E., Urbanec M., Pecháček T., Bakala P., 2016, MNRAS, 457, L19

Van Paradijs J., et al., 1990, A\&A, 234, 181

Van Straaten S., Ford E. C., van der Klis M., Méndez M., Kaaret P., 2000, ApJ, 540, 1049

Wijnands R. A. D., van der Klis M., van Paradijs J., Lewin W. H. G., Lamb F. K., Vaughan B., Kuulkers E., 1997, ApJ, 479, L141

Zdziarski A. A., Johnson W. N., Magdziarz P., 1996, MNRAS, 283, 193

Zhang W., Lapidus I., Swank J. H., White N. E., Titarchuk L., 1997, IAU Circ., 6541

Zhang G., Méndez M., Sanna A., Ribeiro E. M., Gelfand J. D., 2017, MNRAS, 465, 5003

Życki P. T., Done C., Smith D. A., 1999, MNRAS, 309, 561 